\title{
Varied expression and localization of multiple galectins in different cancer cell lines
}

\author{
ARUN SATELLI, PREMA S. RAO, PREM K. GUPTA, PAUL R. LOCKMAN, \\ KALKUNTE S. SRIVENUGOPAL and U. SUBRAHMANYESWARA RAO
}

\begin{abstract}
The Anticancer Resistance Group and the Cancer Biology Center, Department of Pharmaceutical Sciences, Texas Tech University Health Sciences Center, Amarillo, TX 79106, USA
\end{abstract}

Received September 10, 2007; Accepted October 24, 2007

\begin{abstract}
Galectins play a key role in oncogenic processes. Although several galectins are known, their relative expression at the mRNA and protein levels, the subcellular localization, and their relationship to the oncogenic manifestation remains unclear. Herein we report a comprehensive characterization of the expression of major galectins in human breast cancer (drug-sensitive MCF-7 and drug-resistant MCF-7/Adr ${ }^{\mathrm{R}}$ ), colon cancer (HCT-116 and HT-29), and glioma (T98G) cell lines, as these cells are common model systems for studying oncogenic processes. The expected $\sim 14.5 \mathrm{kDa}$ galectin-1, predominantly cytosolic, was detected in the cancer and normal cell lines. Notably, two different molecular forms of galectin-1 with molecular masses of $\sim 13.5$ and $15 \mathrm{kDa}$ were detected in T98G cells, the latter being in the extracellular medium, perhaps a result of post-translational processing. Immunocytochemistry indicated that the extracellular galectin-1 bound to the cell surface was punctated in appearance, suggesting that it was bound to specific receptors. Immunohistological studies indicated that metastasizing carcinomas express high levels of galectin-1. On the other hand, galectin-3 was readily detectable in all cancer cell lines but undetectable in normal cell lines, indicating that galectin-3 is a cancer-specific biomarker protein. Galectin-3 was a cytosolic protein but was not detected in the extracellular medium, indicating that cancer cells do not secrete this galectin. Finally, despite the RT-PCR analysis suggesting the presence of two transcripts of galectin- 8 in all cancer cell lines, the corresponding $\sim 36 \mathrm{kDa}$ protein was only detectable in the nuclear and cytosolic fractions upon cell fractionation. Notably, a different molecular form of galectin-8
\end{abstract}

Correspondence to: Dr U.S. Rao, Department of Pharmaceutical Sciences, School of Pharmacy, SOP Room 409, 1300 Coulter Rd, Amarillo, TX 79106, USA

E-mail: us.rao@ttuhsc.edu

Key words: galectin, breast invasive carcinoma, MCF-7, colon invasive adenocarcinoma, HT-29, T98G, RT-PCR, immunocytochemistry, immunohistochemistry of $\sim 18 \mathrm{kDa}$ was immunoprecipitated from the extracellular media, suggesting that the secreted galectin- 8 undergoes post-translational processing. These results highlight the expression of galectins in different molecular forms in cancers, warranting caution in interpreting the results of functional studies of individual galectins, particularly because these proteins function redundantly in cancer pathways.

\section{Introduction}

Galectins are a family of lectins that are widely expressed in the animal kingdom, ranging from Caenorhabditis elegans to human (1-3). As many as 15 mammalian galectins, termed galectin-1, -2, -3 and others have been identified and characterized in different cancers (3). A closer inspection of the NCBI database indicates that many of these galectins are transcribed as different isoforms, perhaps splice-variants, thus markedly increasing the number of galectins expressed in cells. Galectins are relatively small proteins with molecular masses ranging from 14.5 to $38 \mathrm{kDa}$, and characteristically contain a conserved $\sim 130$ amino acid stretch of carbohydrate recognition domain (CRD) $(4,5)$. Some galectins carry two CRDs (galectin-3, -4, -6, -8, -9 and -12), whereas others contain a single CRD (galectin-1, $-2,-5,-7,-10,-11,-13$ and -14) (6), allowing these lectins to preferentially function as monomers or dimers, respectively. Galectins bind to and cross-link the extracellular matrix components, membrane glycoproteins and lipids present in the adjacent cells through their ability to bind $B$-galactosides present in the carbohydrate moieties, leading to the cell-matrix and cell-cell adhesion (7). Although these observations imply that galectins are extracellular, the amount of galectins secreted in the extracellular surface is minimal (8), and the majority of the expressed galectins is localized in the intracellular space and nucleus (9), indicating that these proteins perform an important intracellular function in physiological and pathological processes.

Among all the known galectins, galectin-1 is wellcharacterized, and is a prototype of the galectin family. In addition to the carbohydrate-binding ability, galectin-1 associates with proteins through protein-protein interactions and participates in a variety of oncogenic processes including transformation and proliferation, cell cycle regulation, cell adhesion, metastasis, inhibition and the promotion of programmed cell death and apoptosis in activated $\mathrm{T}$ cells 
$(10,11)$. Numerous ligands for galectin-1 have been described in different tissues and cells that include actin, laminin, fibronectin, vitronectin, integrins, CA-125, H-ras, CD45 and gemin-4 (6,12-15). Galectin-1 is expressed in a variety of cell types including breast epithelial, thymic epithelial, endothelial and dendritic cells, macrophages, fibroblasts and bone marrow cells $(2,16-20)$. There is an increased galectin- 1 expression in many cancer types including colon, breast, ovary and prostate carcinomas and aggressive gliomas, and an increased accumulation of galectin- 1 in the stroma surrounding tumors in ovarian and prostate carcinoma (2,21-25). Other galectins, specifically galectin-3 and -8 , are involved in a variety of cellular and carcinogenic processes similar to galectin-1. Although this conservation of cellular functions among different galectins is now well recognized, little is known about the expression of different galectins in a specific cancer cell type. In this study, we investigated the expression patterns of major galectins $(-1,-3$ and -8$)$ in the most common breast and colon cancer cells. Our results show that although galectins are widely expressed in human cancers, they exhibit unique expression characteristics, molecular forms and subcellular localization.

\section{Materials and methods}

Materials. Recombinant galectin-1, -3 and -8 and the corresponding antibodies were purchased from R\&D Biosystems, Minneapolis, MN. AlexaFlour-488 coupled secondary antibodies were obtained from Invitrogen, CA. P-glycoprotein (Pgp)-specific UIC2 antibody was obtained from Chemicon International, Temecula, CA. The Pgp-specific $\mathrm{NH}_{2} 11$ antibody was prepared in our laboratory (26). SDS-PAGE and the immunoblotting reagents were purchased from Bio-Rad. Precast SDS-PAGE gels were purchased from Pierce. The oligonucleotides used in this study were synthesized at Integrated DNA Technologies, Inc., Coralville, IA. All other reagents were of analytical grade.

Cell lines and culture conditions. The cell lines (American Type Culture Collection, Vanassas, MD) used in this study were cultured in a $37^{\circ} \mathrm{C}$ humidified incubator with $5 \% \mathrm{CO}_{2}$ in the laboratory as recommended by the supplier. The drugsensitive human breast cancer MCF-7 cells, adriamycinresistant breast cancer cell line, $\mathrm{MCF}-7 / \mathrm{Adr}^{\mathrm{R}}$, human embryonic kidney HEK-293 and the human foreskin fibroblast (HFF-2) cells were cultured in DMEM. The human glioma cell line T98G was cultured in MEM. The human colon adenocarcinoma cell line HT-29 and the human colon carcinoma cell line HCT-116 were cultured in McCoy's 5A medium. The growth media used in this study were supplemented with 10\% FBS and antibiotics (penicillin and streptomycin, $100 \mu \mathrm{g} / \mathrm{ml})$.

Reverse transcription-PCR (RT-PCR). The isolation and detection of different galectin transcripts in cells were carried out by the standard RT-PCR procedures. Briefly, total RNA was extracted using a Trizol reagent (Invitrogen, CA) from different cells and then subjected to RT-PCR as previously described (26), using gene-specific primers. Full-length galectins were amplified using the following primer pairs: galectin-1: forward primer, 5'-ATGGCTTGTGGTCTGGTC GCC-3' and reverse primer, 5'-TCAGTCAAAGGCCACAC ATTTGA-3'; galectin-3: forward primer, 5'-ATGGCAGACA ATTTTTCGCTCC-3' and reverse primer, 5'-TTATATCATG GTATATGAAGCA-3'; galectin-4: forward primer, 5'-ATG TCTGTTTACATCCAAGGAGTG-3' and reverse primer, 5'-TTAGATCTGGACATAGGACAAGGTGACAT-3'; galectin-7: forward primer, 5'-ATGTCCAACGTCCCCC ACAAGTC-3' and reverse primer, 5'-TCAGAAGATCCTC ACGGAGTCCA-3' and galectin-8: forward primer, 5'-ATG TTGTCCTTAAACAACCTACAG-3' and reverse primer, 5'CTACCAGCTCCTTACTTCCAG-3'. The amplification of GAPDH was carried out using the primers 5'-CAGCCGAGC CACATCG-3' and 5'-TGAGGCTGTTGTCATACTTCTC-3'. The PCR amplified DNA fragments were separated on $1 \%$ agarose gel containing ethidium bromide and visualized using the Bio-Rad ChemiDoc system. The amplified DNA fragments were subsequently sub-cloned in the pGEMT easy vector (Promega, CA) and the insert DNA was sequenced at the DNA sequencing facility of the University of Nebraska, Lincoln, NE.

Immunocytochemistry. Immunocytochemical detection of galectins in different cancer cells was performed according to the method previously described (26) with some modification. Briefly, to detect the intracellular galectins, cells growing on coverslips were fixed with $1 \%$ paraformaldehyde and then permeabilized in buffer containing $0.25 \%$ Nonidet P40. Cells were then incubated with galectin-specific primary antibody as indicated in the text, followed by staining with the relevant AlexaFluor-488 coupled secondary antibody. To immunostain the extracellularly located galectins, cells were plated on coverslips placed in 12-well plates and grown to $\sim 50 \%$ confluency. Cells were washed gently once with PBS, incubated with fresh growth medium supplemented with a specific primary antibody and incubation was continued in the $37^{\circ} \mathrm{C}$ incubator for $45 \mathrm{~min}$. The cells were then washed in PBS and fixed in $1 \%$ paraformaldehyde and immunostained using the relevant AlexFluor-488 coupled secondary antibody. The cellular location of AlexaFluor-488 fluorescence in cells was determined using a Leica laser confocal microscope (TCS SL, Leica Microsystems GmbH, Heidelberg), with a $63 \times 1.4$ oil objective and immersion oil, and the images were digitally recorded on a computer hard drive.

Immunohistochemistry. The paraffin-embedded human breast invasive carcinoma and colon invasive adenocarcinoma sections were obtained from the Department of Pathology, University of Nebraska Medical Center, Omaha, NE. These tissue sections were immunostained using anti-galectin antibodies in conjunction with AlexaFluor-488-conjugated secondary antibodies according to the procedures previously described (26). Specimens were observed under an Olympus IX81 microscope and the images were digitally recorded on a computer hard drive.

Immunoprecipitation. To detect secreted galectins, T98G cells were grown to $\sim 80 \%$ confluence in $100-\mathrm{mm}$ tissue culture dishes and the spent medium was collected. As a control, the fresh growth medium was used. Galectins were immuno- 


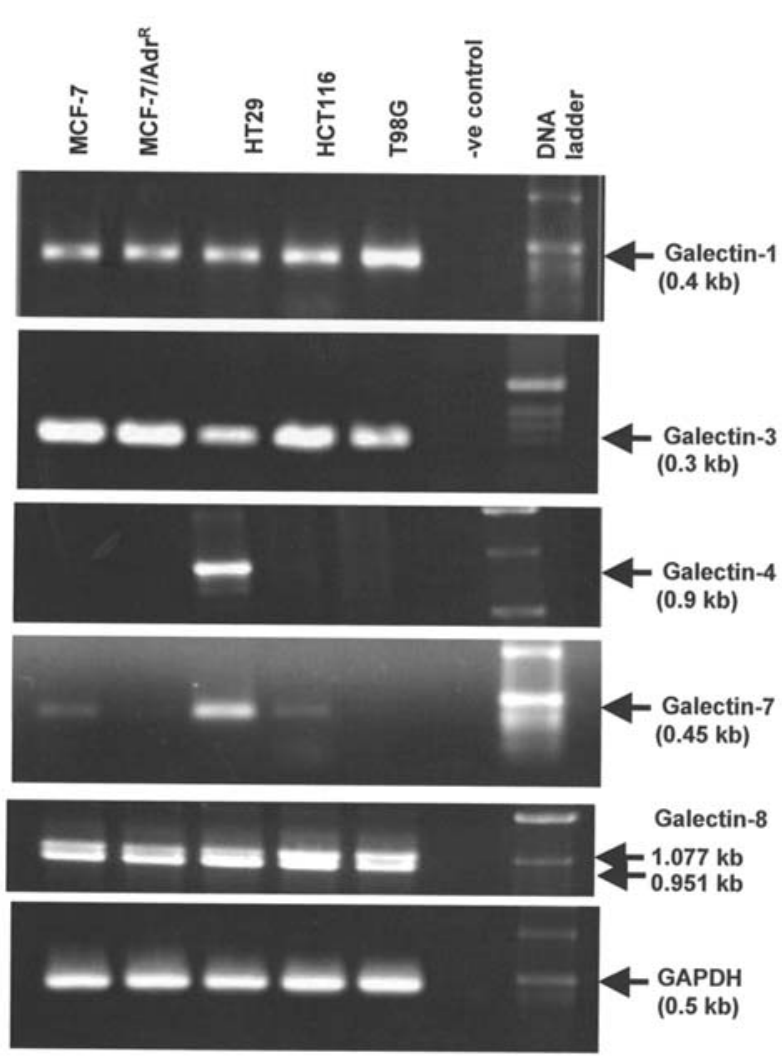

Figure 1. The RT-PCR analysis of various galectin transcripts in different cancer cell lines. Three out of $50 \mu 1$ of the reverse transcription reaction mixtures obtained from $5 \mu \mathrm{g}$ of total RNA isolated from each cell line was used as a template in the PCR. Each of the indicated galectin cDNA was amplified using its specific primer set mentioned in Materials and methods. Glyceraldehyde-3-phosphate dehydrogenase (GAPDH) was amplified to serve as an internal control for PCR. Equal volumes of these PCR mixtures were separated on $1 \%$ agarose gel containing ethidium bromide, and the images were digitally captured by the Bio-Rad ChemiDoc System. The expected size of each galectin by using the primer set is indicated.

precipitated using specific antibodies according to the procedure of Rao et al (26). The immunoprecipitates were subjected to SDS-PAGE followed by immunoblotting for the detection of galectins.

The isolation of nuclear and cytosolic fractions. Cells grown to $\sim 80 \%$ confluency in a $775 \mathrm{~cm}^{2}$ flask were collected in cold PBS, centrifuged at $1000 \mathrm{x} \mathrm{g}$ and the pellet obtained was incubated in $0.5 \mathrm{ml}$ lysis buffer containing $1 \mathrm{X}$ PBS, $0.1 \%$ Triton X-100, 0.1\% Nonidet P40, $0.2 \mathrm{mM}$ DTT, $25 \mathrm{mM} \mathrm{NaF}$, $5 \mathrm{mM}$ sodium vanadate, $0.1 \mathrm{mM} \mathrm{ZnCl}_{2}, 20 \%$ glycerol and protease inhibitor cocktail (Sigma) on ice for $10 \mathrm{~min}$ with intermittent pipetting. The suspension was centrifuged at $1000 \mathrm{x} g$ for $15 \mathrm{~min}$. The supernatant obtained was then centrifuged at $60,000 \mathrm{x} \mathrm{g}$ and the clear supernatant termed cytosolic fraction was collected. The pellet, obtained from the above $1000 \mathrm{x}$ g centrifugation contained nuclei, was washed once in the above lysis buffer without detergents, re-suspended and then sonicated for $30 \mathrm{sec}$. The sonicate was centrifuged at $60,000 \times \mathrm{g}$ and the supernatant obtained was termed a nuclear fraction. The cytosolic and nuclear fractions thus obtained were analyzed for the presence of galectins by immunoblotting.
SDS-PAGE and immunoblotting. The cells growing in the tissue culture dishes were scraped into the growth medium and centrifuged at $2000 \mathrm{rpm}$ for $5 \mathrm{~min}$ in a microcentrifuge. The pelleted cells were washed once with ice-cold PBS, and the cellular proteins were precipitated with $6 \%(\mathrm{w} / \mathrm{v})$ trichloroacetic acid. The precipitated proteins were dissolved in Laemmli buffer. SDS-PAGE, the transfer of proteins onto the PVDF membranes and immunoblotting were performed as previously described (26).

Protein estimation. The protein content in the samples was estimated by the Bio-Rad DC protein assay kit using bovine serum albumin as standard.

Experiments. Each of the experiments in this study was carried out a minimum of three times using different batches of cell lines with essentially identical results.

\section{Results}

RT-PCR analysis of galectins in different cancer cell lines. We examined the expression of different galectins in several established cell lines using the RT-PCR procedure. Total RNA $(5 \mu \mathrm{g})$ isolated from each cell line was used in the reverse transcription. The resulting reaction mixtures were used as template DNAs in the PCR to obtain a quantitative analysis of the gene expression. Representative amplified DNA fragments were subcloned into a pGEMT easy cloning vector and sequenced to confirm their identity. Fig. 1 shows the amplification of $\sim 0.4 \mathrm{~kb}$ galectin- 1 and $0.3 \mathrm{~kb}$ galectin- 3 cDNA, which were present in nearly similar amounts in all of the cancer cell lines. Galectin-4 cDNA was readily amplified from HT-29 cells but was not detectable in other cell lines, perhaps due to a low abundance. Although all of the cancer cell lines contained a galectin-7 transcript, the expression levels among the cell lines varied. Finally, the galectin-8specific primers used in the PCR amplified two transcripts of 1077 and $951 \mathrm{bp}$ in all of the cancer cell lines, and their amounts were nearly equal. DNA sequencing and alignment with the NCBI database using a BLAST server identified these two DNA fragments as galectin-8 isoform 1 (NM_ 201543) and 2 (NM_201544), respectively.

To evaluate the expression levels of different galectins in each cell line, the RT-PCR mixtures of the different galectins prepared as above were separated by agarose gel electrophoresis. Fig. 2 shows the relative levels of different galectins in the cancer cell lines. The drug-sensitive MCF-7 and the drugresistant $\mathrm{MCF}-7 / \mathrm{Adr}^{\mathrm{R}}$ cells contained galectin-1, -3 and -8 in significantly higher amounts when compared to the levels of galectin-4 and -7 . The HT-29 cell line uniquely contained high levels of galectins- 4 and -7 when compared to other cell lines. Galectins- 4 and -7 were less abundant in HCT-116 and T98G cells. As controls, we analyzed the expression of these galectins in the human embryonic kidney HEK-293 and fibroblast HFF-2 cells using the identical RT-PCR condition and the results showed that these non-cancer cells transcribe galectins- 1 and -3 but not other forms. Together, these results suggested that galectins are differentially transcribed in cancer cells and galectins- $4,-7$ and -8 are cancer-specific, but absent in the non-cancer cell lines. 


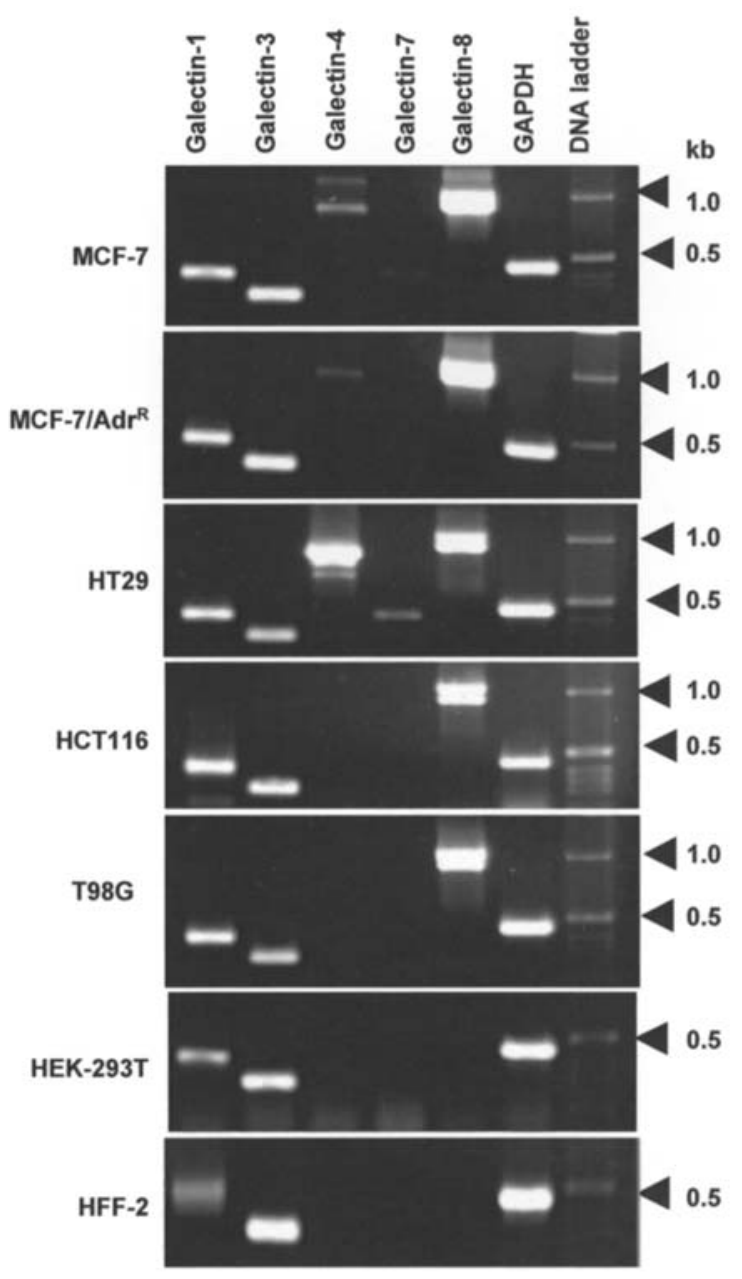

Figure 2. A comparative analysis of various galectins showing their relative amounts in different cancer cell lines. The RT-PCR mixtures of different galectins obtained from each cancer cell line shown in Fig. 1 were separated on $1 \%$ agarose gel electrophoresis and the images were digitally captured using the Bio-Rad ChemiDoc system. The sizes of the standard DNA fragments in the DNA ladder are indicated with arrows.

Immunoblot analysis of galectins. To further determine the expression of galectins in the above cells, we analyzed the expression of galectins-1, -3 and -8 , because recent studies indicated that these lectins play a key role in oncogenic processes (27-29). Fig. 3 shows that MCF-7, MCF-7/Adr ${ }^{\mathrm{R}}$ and HCT-116 cells contained the expected $14.5 \mathrm{kDa}$ galectin- 1 in nearly equal amounts, corroborating the above RT-PCR studies. Of note are the significantly high levels of galectin-1 expression and its resolution into two species with molecular masses of $\sim 14.5$ and $13.5 \mathrm{kDa}$ in T98G and HFF-2 cells, despite the similar levels of steady state gene transcripts in these cells versus that of other cell lines. This suggests that either the half-life or efficiency of the translation of galectin-1 in T98G and HFF-2 cells is higher. On the other hand, galectin-1 was not detected in HEK-293 cells, although these cells contained significant levels of transcript, perhaps indicating the existence of translational repression mechanisms in these cells.

As expected from the transcription levels seen in Figs. 1 and 2 , the $\sim 30 \mathrm{kDa}$ galectin-3 was detectable in all of the cancer cell lines at relatively similar amounts except for

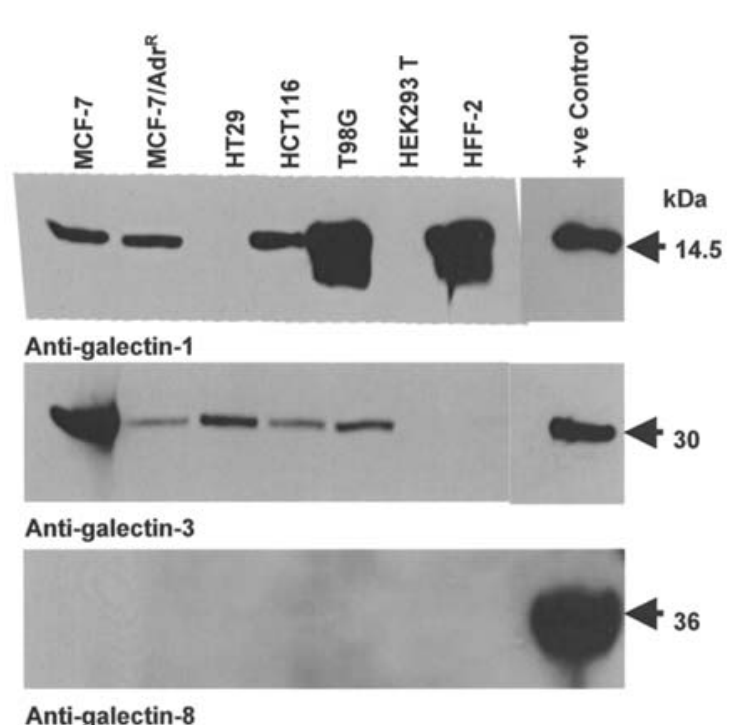

Figure 3. A Western blot analysis of the galectin expression in different cancer cell lines. Lysates (10 $\mu \mathrm{g}$ total cellular protein each) from MCF-7, MCF-7/Adr ${ }^{\mathrm{R}}$, HT-29, HCT-116, T98G, HEK-293 and HFF-2 cells were separated on 4-20\% acrylamide gradient SDS-PAGE gels followed by immunoblotting using anti-galectin- $1,-3$ and -8 antibodies. Recombinant galectin proteins separated on these gels (lane, +ve control), are indicated with arrows. Their molecular masses are also shown. No other proteins in the entire blot are immunoreactive, thus only a portion where the proteins are detected is shown.

MCF-7 cells. However, galectin-3 was not detectable in either HEK-293 or HFF-2 cells, despite the presence of corresponding mRNA. Finally, immunoblotting using monoclonal antibody to galectin- 8 or the polyclonal antibodies did not indicate the presence of $\sim 36 \mathrm{kDa}$ galectin- 8 in any of the cell lines, even after extended chemiluminescence. Since the recombinant galectin- 8 was easily immunoreactive in these blots, the results suggest the absence of the galectin- 8 protein in any of the cell lines. From this analysis it is apparent that cancer cells exhibit a fine control of lectin translation, and it is therefore essential to combine the RT-PCR analysis with immunoblotting profiles to assess the function of the individual galectins.

The cellular localization of galectins. Galectins are known to be localized in the cytosol, nucleus and in the extracellular space. Therefore, we characterized the subcellular location of galectins in T98G cells, which was used as a model for this analysis. T98G cells grown to $\sim 80 \%$ confluency were fractionated into cytosol and nuclear fractions, and then analyzed for the presence of different galectins by immunoblotting, as described in Materials and methods. Fig. 4 shows that galectin- 1 and -3 were present in the cytosolic but absent in the nuclear fraction. Notably, when the subcellular fractions were analyzed for the presence of galectin-8, the $\sim 36 \mathrm{kDa}$ protein was predominantly detected in the nuclear fraction, and to some extent in the cytosolic. The reasons for our failure to detect galectin- 8 in the whole cell lysates by direct SDSPAGE/immunoblotting analysis (Fig. 3) are unclear at present. The identification of galectin- 8 in the cytosolic and nuclear fractions indicates that a cell fractionation procedure is a prerequisite for the detection of galectin- 8 . 

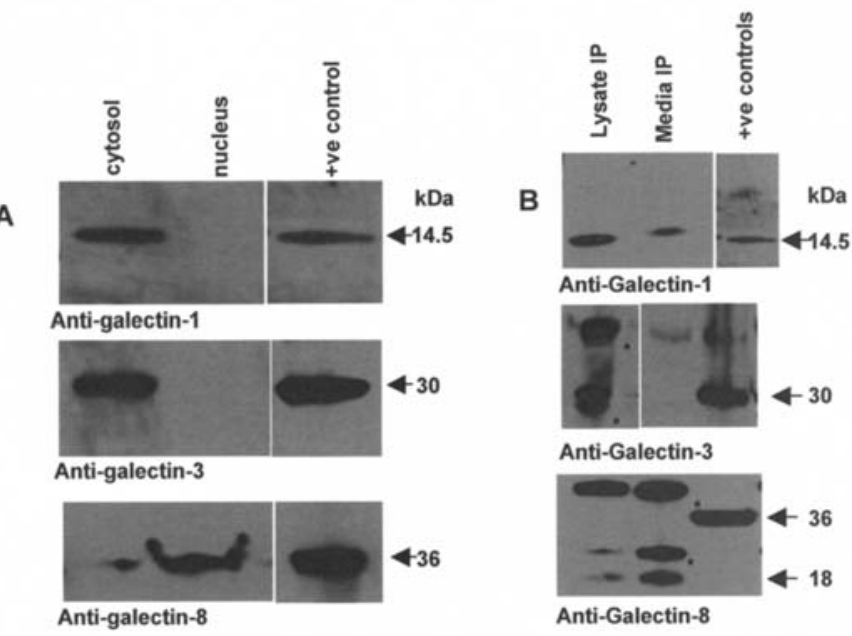

Figure 4. (A) Sub-cellular fractionation of galectins-1, -3 and -8 from T98G cells grown to $\sim 80 \%$ confluency in $10-\mathrm{cm}$ culture dishes and fractionated into nuclear and cytosolic fractions using lysis buffer as described in Materials and methods. The fractions were then analyzed for galectin-1, -3 and -8 by immunoblotting using the indicated antibodies. Recombinant galectin proteins separated on these gels (lane, +ve control), are indicated with arrows. Their molecular masses are also shown. (B) The immunoprecipitation of intra- and extracellular galectins-1, -3 and -8 from T98G cells which were grown to $\sim 80 \%$ confluency. The cells and the spent medium were collected and galectins- $1,-3$ and -8 were immunoprecipitated from the cell lysates and the extracellular medium using anti-galectin antibodies as described in Materials and methods. The immunoprecipitates were then analyzed by immunoblotting. The 50 and $25 \mathrm{kDa}$ immunoglobulin bands are indicated with ${ }^{*}$. Recombinant galectin proteins separated on these gels (lane, +ve control), are indicated with arrows and their molecular masses are also shown. The immunoprecipitated $\sim 18 \mathrm{kDa}$ galectin- 8 is indicated with an arrow.

To determine whether galectins are secreted in the extracellular space, T98G cells were grown to $\sim 80 \%$ confluency and the spent medium was collected. Galectins in the spent medium were immunoprecipitated using anti-galectin antibodies as described in Materials and methods. Fig. 4 also shows that a significant amount of $\sim 15 \mathrm{kDa}$ protein immunoreactive with anti-galectin-1 was detectable in the spent medium, which is slightly higher in molecular mass, compared to the size of the galectin-1 immunoprecipitated from the total cell lysates. Since no protein was immunoprecipitated by anti-galectin-1 from the fresh growth medium (not shown), the $\sim 15 \mathrm{kDa}$ protein is either a different or post-translationally modified form of galectin-1. On the other hand, the $30 \mathrm{kDa}$ galectin-3 was not detectable in the immunoprecipitates obtained from the spent medium, which was, however, immunoprecipitated from the cell lysates. Although the $\sim 36 \mathrm{kDa}$ galectin-8 was not detected in the immunoprecipitates prepared either from the cell lysates or the spent growth medium, an $\sim 18 \mathrm{kDa}$ anti-galectin- 8 immunoreactive protein was consistently detectable in the cell lysates and extracellular medium. The identity of this $\sim 18 \mathrm{kDa}$ protein is unclear at present (see Discussion). These results together suggest that the intracellular galectins-1, -3 and -8 have the expected molecular masses. Since galectins detected in the extracellular medium are different from that of the expected ones, it is likely that the secreted galectins undergo further modification before or after secretion.

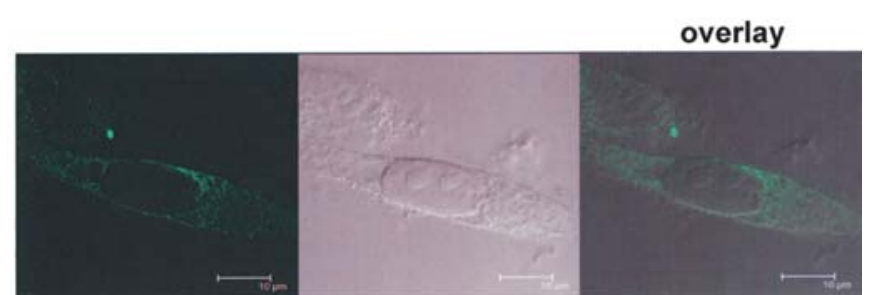

\section{Galectin-1}

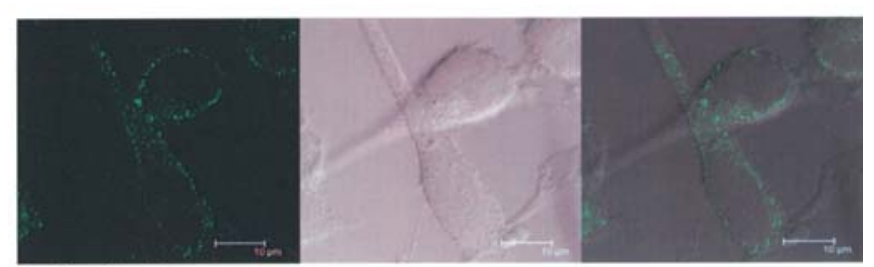

Galectin-3

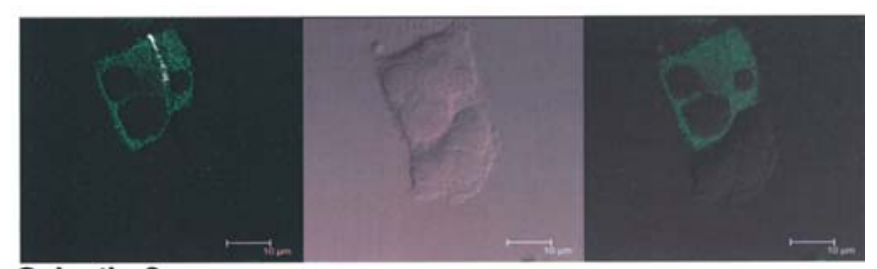

Galectin-8

Figure 5. The immunocytochemistry of galectins-1, -3 and -8 expression and T98G cells. Cells grown on coverslips were fixed in paraformaldehyde, immunostained with the anti-galectin antibodies, and then the antibody bound regions were probed by incubating the cells with the AlexaFluor-488 coupled secondary antibody as described in Materials and methods. The green flourescence of AlexaFluor-488 was recorded using a Leica confocal laser microscope.

The immunocytochemistry of galectins. To further characterize the cellular localization of galectins- $1,-3$ and -8 , T98G cells were grown on coverslips and fixed using paraformaldehyde. Cells were immunostained with anti-galectin antibodies in conjunction with AlexaFluor-488-coupled secondary antibody and then analyzed by confocal laser microscopy, as described in Materials and methods. Fig. 5 shows that the AlexaFluor-488 fluorescence associated with galectins- 1 and -3 was predominantly cytosolic and absent in the nucleus, supporting the results shown in Fig. 4. The staining of cells with anti-galectin- 8 indicated the presence of galectin- 8 in different locations of different cells. Certain cells contained galectin-8 exclusively in the cytosol, whereas other cells contained immunofluorescence in the nuclei and cytosol. Notably, some cells did not immunostain at all suggesting the absence of the galectin- 8 expression. Although the reasons for the complete absence of galectin- 8 in some cells and its differential subcellular distribution in different cells of the same cell line are unclear, it is likely that the expression of galectin- 8 is finely regulated and appears cell cycle stagedependent.

Since galectins are secreted in the extracellular space, it is likely that these lectins are bound to the carbohydrate moieties of the cell surface proteins and lipids. Thus, we tested the possibility that galectin-1 is bound to the extracellular surface by the in vivo labeling of $\mathrm{MCF}-7 / \mathrm{Adr}^{\mathrm{R}}$ cells. Pgpexpressing $\mathrm{MCF}-7 / \mathrm{Adr}^{\mathrm{R}}$ cells growing on coverslips were incubated with anti-galectin- 1 at $37^{\circ} \mathrm{C}$ for $45 \mathrm{~min}$. The cells 

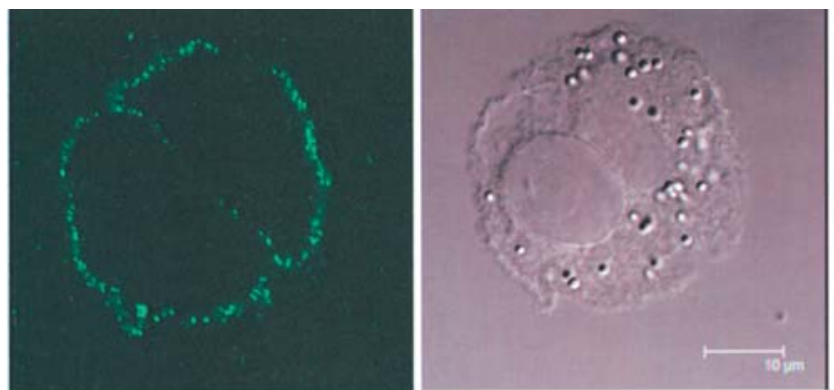

\section{Anti-galectin-1}
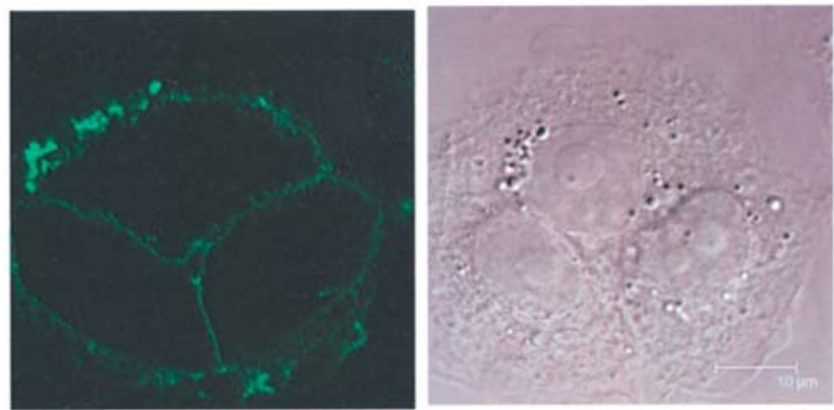

Anti-Pgp (UIC-2)
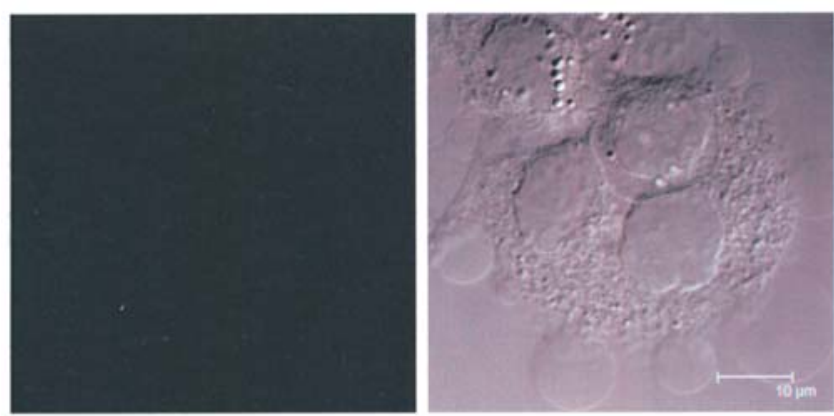

Anti-Pgp $\left(\mathrm{NH}_{2} 11\right)$

Figure 6. In vivo labeling of the cell surface of galectin-1 and Pgp. The $\mathrm{MCF}-77 / \mathrm{Adr}^{\mathrm{R}}$ cells growing on the coverslips were incubated with antigalectin- 1 antibody for $45 \mathrm{~min}$, fixed and then stained with AlexaFluor-488 coupled secondary antibody. Similarly, the plasma membrane-bound Pgp in the MCF7/Adr ${ }^{\mathrm{R}}$ cells was labeled with UIC2 and $\mathrm{NH}_{2} 11$ antibodies in conjunction with AlexaFluor-488 coupled secondary antibody. The cells were subsequently fixed in paraformaldehyde and labeled with the secondary antibody. The green fluorescence associated with the AlexaFluor-488 was recorded using a Leica confocal laser microscope.

were fixed in buffer containing paraformaldehyde but lacking detergents and then immunostained with anti-galectin- 1 in conjunction with AlexaFluor-488 coupled secondary antibody. Fig. 6 shows that AlexaFluor-488 immunofluorescence was predominantly localized at the cell surface of the MCF-7/Adr ${ }^{\mathrm{R}}$ cells, suggesting that these cells secrete galectin-1, which was bound to the cell surface. To determine whether the in vivo labeling methodology used here detects only the extracellular galectin-1, we immunostained these cells for Pgp, a plasma membrane protein, using two wellcharacterized antibodies, UIC2 and $\mathrm{NH}_{2} 11$. The UIC2 antibody is known as a conformation-specific antibody that binds to the extracellular epitope (30), whereas the $\mathrm{NH}_{2} 11$ antibody recognizes an epitope in Pgp that is intracellularly located $(26,31)$. Thus, the MCF-7/Adr ${ }^{\mathrm{R}}$ cells growing on the

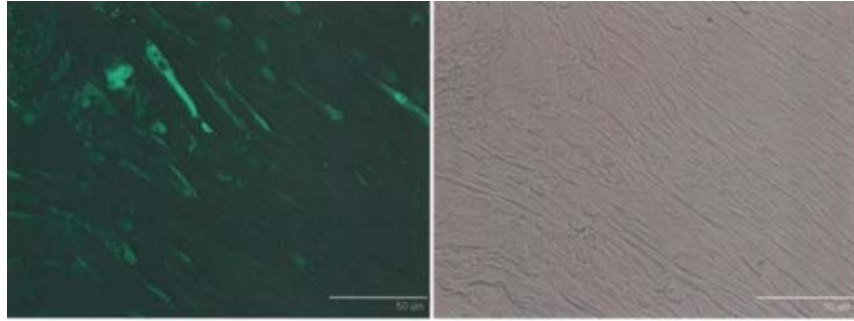

Breast invasive carcinoma

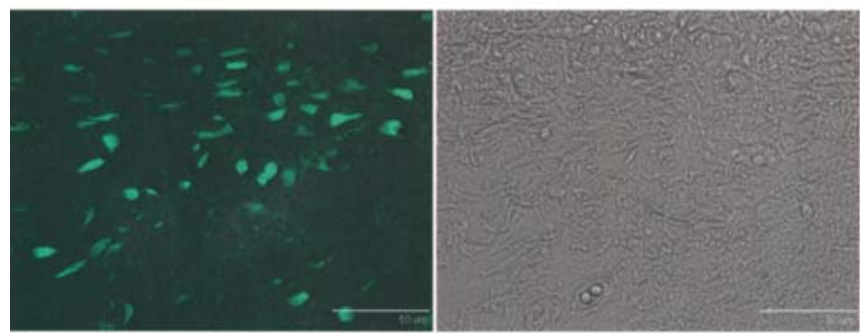

Colon invasive adenocarcinoma

Figure 7. Immunohistochemistry of galectin-1 in human breast invasive carcinoma and colon invasive adenocarcinoma Paraffin-embedded sections from the primary specimens of the human breast invasive carcinoma and colon invasive adenocarcinoma were stained for galectin-1 using antigalectin-1 antibody in conjunction with AlexaFluor-488 coupled antibodies. The AlexaFluor-488 fluorescence was detected using an Olympus IX81 fluorescence microscope. Images were recorded on the computer hardware. The bar is $50 \mu \mathrm{m}$. The tumor cells are intensely fluorescent.

coverslips were immunostained with UIC2 and $\mathrm{NH}_{2} 11$ antibodies for $45 \mathrm{~min}$ as described above, and the location of these antibodies was determined by confocal laser microscopy analysis. Fig. 6 shows that UIC2-bound immunofluorescence was present exclusively at the cell surface. On the other hand, no immunofluorescence was detectable when $\mathrm{NH}_{2} 11$ was used under identical experimental conditions. We have previously shown that the immunostaining of Pgp with $\mathrm{NH}_{2} 11$ requires a prior permeabilization of cells with detergents (26), and failure to immunostain Pgp with $\mathrm{NH}_{2} 11$ in the absence of detergents, suggested that immunostaining procedures used in this study selectively immunostain protein epitopes present at the exposed cell surface. These data also indicate that the antibodies are not internalized. Taken together, these results suggested that the cancer cells secrete galectin-1, which is bound to the extracellular surface.

Immunohistochemistry of galectin-1. To further determine the expression of galectins in cancers, the paraffin-embedded sections from primary specimens of the human breast invasive carcinoma and colon invasive adenocarcinoma were stained for galectin-1 using the respective antibodies in conjunction with AlexaFluor-488 coupled secondary antibodies. The results are shown in Fig. 7. Galectin-1 immunostaining was predominantly associated with the tumor cells, and their identity was confirmed by hematoxylin-eosin staining (not shown). Using similar experimental procedures, we analyzed the expression of galectin-3 in these carcinomas, which indicated that the these invasive carcinomas contain elevated levels of galectin-3 (not shown). Taken together these results suggested that elevated levels of galectins are a characteristic of metastatic tumor cells. 


\section{Discussion}

Galectins are a family of proteins that bind to $\beta$-galactosidecontaining oligosaccharides and proteins leading to their multipotent intra- and extracellular biological functions. Galectins are involved in several cellular processes including tumor cell adhesion and migration, tumor aggressiveness, modulation of apoptosis (32), and an immune response through the induction of apoptosis in activated T-cells (10), highlighting their importance as cancer molecular targets. Although 15 galectins are known, expression profiles and functions of only a few galectins are characterized in different cell lines $(33,34)$, perhaps due to their abundance and importance in cancers. The general notion resulting from these studies is that galectins as a whole share functional characteristics, influenced by the nature of the cell and cell cycle stage. Since the above-mentioned functions are exhibited equipotently by many different galectins, it is necessary to establish the expression profiles of all galectins in the same tissues or cell lines, prior to studies aimed at establishing their specific function. Lahm and co-workers (35) have analyzed the expression of several galectins in numerous cancer-derived cell lines by RT-PCR, establishing a database useful for galectin biology. Importantly, these studies have demonstrated that galectin expression profiles are variable not only in cell lines derived from different origins, but also in cells derived from the similar tissues/tumors, necessitating a fresh look at their expression patterns in experimental models.

In our efforts to unravel the roles of galectins in cancer drug resistance, we characterized the expression of galectins in many cancer cell lines and a few primary tumor specimens. The data presented here indicate that galectins are differentially transcribed and expressed in cancer cell lines, corroborating the studies of Lahm and co-workers (35). While the RT-PCR analysis indicated that galectin-1 is actively transcribed in all cell lines, its translated protein was not detectable in HT29 and HEK-293 cells. Since HEK-293 are normal and epithelial in origin, the absence of galectin-1 in this cell line and its presence in cancer cells such as MCF-7 and HCT-116, which are also of epithelial origin, it appears possible that galectin-1 is up-regulated in epithelial cancers. In contrast, the observation that the cancer cell line T98G and the normal cell line HFF-2 cells, which are fibroblast in origin, contain high levels of galectin-1, suggests that galectin-1 is constitutively expressed in fibroblast-derived cancers.

Although the expected galectin-1 form with a molecular mass of $\sim 14.5 \mathrm{kDa}$ was detected in all cell lines, we also detected an unexpected $\sim 13.5 \mathrm{kDa}$ galectin-1 form in T98G and HFF-2 cells. However, the molecular nature of this form or its function is unclear at present. Notably, the migration of this $\sim 13.5 \mathrm{kDa}$ galectin- 1 is similar to a galectin-1ß form characterized by Miura et al (36), which lacks the first six amino acids and is involved in axonal regeneration. Whether the observed $\sim 13.5 \mathrm{kDa}$ galectin- 1 in T98G and HFF-2 cells is galectin-1ß or an entirely different species needs to be established. The immunoprecipitates of the extracellular medium contained a galectin-1 form with a molecular mass of $\sim 15 \mathrm{kDa}$, which is slightly larger than the expected mass. In view of the uncertainty of the secretory pathway involved in the galectin-1 secretion, as it lacks the required signal peptide, the higher molecular mass of galectin-1 suggests that a specific post-translational modification resulting in the apparently high molecular mass is a prerequisite for galectin-1 export to the extracellular milieu. The immunocytochemical analysis (Fig. 5) suggests that galectin-1 bound to the cell surface is particulate and present in a restrictive manner at specific locations of the plasma membrane, unlike the uniform distribution of P-glycoprotein on the plasma membrane (Fig. 6). This observation suggests that the $\sim 15 \mathrm{kDa}$ extracellular galectin-1 binds to specific cell surface receptors, which are involved in the effects elicited by the extracellular galectin. The identification of the plasma membrane-bound receptor to which galectin-1 binds will allow for a better understanding of the functional significance of this secreted galectin-1 form.

Our studies also provided some insight into the dissimilarities of the galectin- 3 expression in cancer and normal cells. HEK-293 and HFF-2, two non-cancer cell lines did not express galectin-3 despite their ability to transcribe genes, whereas this galectin species showed ubiquitous distribution in all cancer cells. These data suggest that galectin-3 may be useful as a cancer cell biomarker. The presence of high levels of galectin-3 in drug-sensitive MCF-7 cells when compared to its drug-resistant counterpart MCF-7/ $\mathrm{Adr}^{\mathrm{R}}$ cells, suggests that galectin-3 plays a role in the modulation of cancer drug resistance. Moreover, galectin-3 is obviously a cytosolic protein and was not secreted in the extracellular medium by $\mathrm{T} 98 \mathrm{G}$ cells, as judged by the immunoprecipitation of this galectin form from the extracellular medium. These results were corroborated by the immunocytochemical studies performed similarly in the detection of extracellular galectin-1, which clearly indicated the absence of extracellular galectin-3 (not shown).

Finally, although galectin- 8 is abundantly transcribed in all of the cell lines tested, our attempts to detect galectin- 8 in whole cell lysates were unsuccessful. However, its detection i) after subcellular fractionation, ii) by immunocytochemistry, albeit, not in all T98G cells and, iii) the presence of an $\sim 18 \mathrm{kDa}$ anti-galectin- 8 antibody reactive protein in the extracellular medium, all point to its high level of regulation and its possible expression at specific cell cycle phases. Notably, the NCBI database has an additional splice variant of galectin-8 (accession number: CAI13767), whose predicted molecular mass is also $\sim 18 \mathrm{kDa}$. Galectins -4 and -7 are present in low abundance, except that HT29 cells contain a significantly higher amount of galectin-4. These findings highlight the need to perform RT-PCR and immunoblotting in galectin gene expression analyses.

In conclusion, our findings suggest that cancer cells transcribe and translate the known galectins differentially, which exist in different molecular species. A further understanding of these new molecular forms of galectins in oncogenic processes is necessary to facilitate new strategies for anticancer therapy.

\section{Acknowledgements}

This work was supported by grants from the Department of Defense (BC046411), the National Institute of Health (CA106625) and the Women's Health Research Institute, 
TTUHSC to U.S. Rao. We thank Dr Linda Baum for kindly providing us with the galectin-1 cDNA.

\section{References}

1. Toscano MA, Ilarregui JM, Bianco GA, Campagna L, Croci DO, Salatino M and Rabinovich GA: Dissecting the pathophysiologic role of endogenous lectins: Glycan-binding proteins with cytokine-like activity? Cytokine Growth Factor Rev 18: 57-71, 2007.

2. Lahm H, Andre S, Hoeflich A, Kaltner H, Siebert HC, Sordat B, von der Lieth $\mathrm{CW}$, Wolf $\mathrm{E}$ and Gabius HJ: Tumor galectinology: Insights into the complex network of a family of endogenous lectins. Glycoconj J 20: 227-238, 2004.

3. van den Brule F, Califice S and Castronovo V: Expression of galectins in cancer: A critical review. Glycoconj J 19: 537-542, 2004.

4. Barondes SH, Castronovo V, Cooper DN, Cummings RD, Drickamer K, Feizi T, Gitt MA, Hirabayashi J, Hughes C, Kasai K, et al: Galectins: a family of animal beta-galactosidebinding lectins. Cell 76: 597-598, 1994.

5. Perillo NL, Marcus ME and Baum LG: Galectins: versatile modulators of cell adhesion, cell proliferation, and cell death. J Mol Med 76: 402-412, 1998.

6. Elola MT, Chiesa ME, Alberti AF, Mordoh J and Fink NE: Galectin-1 receptors in different cell types. J Biomed Sci 12: 13-29, 2005.

7. Ozeki Y, Matsui T, Yamamoto Y, Funahashi M, Hamako J and Titani K: Tissue fibronectin is an endogenous ligand for galectin-1. Glycobiology 5: 255-261, 1995 .

8. Cho $\mathrm{M}$ and Cummings RD: Galectin-1, a beta-galactosidebinding lectin in Chinese hamster ovary cells. II. Localization and biosynthesis. J Biol Chem 270: 5207-5212, 1995.

9. Moutsatsos IK, Wade M, Schindler M and Wang JL: Endogenous lectins from cultured cells: nuclear localization of carbohydrate-binding protein 35 in proliferating 3T3 fibroblasts. Proc Natl Acad Sci USA 84: 6452-6456, 1987.

10. Perillo NL, Pace KE, Seilhamer JJ and Baum LG: Apoptosis of T cells mediated by galectin-1. Nature 378: 736-739, 1995.

11. Matarrese P, Tinari A, Mormone E, Bianco GA, Toscano MA, Ascione B, Rabinovich GA and Malorni W: Galectin-1 sensitizes resting human T lymphocytes to Fas (CD95)mediated cell death via mitochondrial hyperpolarization, budding, and fission. J Biol Chem 280: 6969-6985, 2005.

12. Camby I, Le Mercier M, Lefranc F and Kiss R: Galectin-1: a small protein with major functions. Glycobiology 16: 137R-157R, 2006.

13. Paret C, Bourouba M, Beer A, Miyazaki K, Schnolzer M, Fiedler $\mathrm{S}$ and Zoller M: Ly6 family member $\mathrm{C} 4.4 \mathrm{~A}$ binds laminins 1 and 5, associates with galectin-3 and supports cell migration. Int J Cancer 115: 724-733, 2005.

14. Horiguchi N, Arimoto K, Mizutani A, Endo-Ichikawa Y, Nakada $\mathrm{H}$ and Taketani S: Galectin-1 induces cell adhesion to the extracellular matrix and apoptosis of non-adherent human colon cancer Colo201 cells. J Biochem 134: 869-874, 2003.

15. Seelenmeyer C, Wegehingel S, Lechner J and Nickel W: The cancer antigen CA125 represents a novel counter receptor for galectin-1. J Cell Sci 116: 1305-1318, 2003.

16. Brinck U, Bosbach R, Korabiowska M, Schauer A and Gabius HJ: Histochemical study of expression of lectin-reactive carbohydrate epitopes and glycoligand-binding sites in normal human appendix vermiformis, colonic mucosa, acute appendicitis and colonic adenoma. Histol Histopathol 11: 919-930, 1996.

17. Berberat PO, Friess H, Wang L, Zhu Z, Bley T, Frigeri L, Zimmermann A and Buchler MW: Comparative analysis of galectins in primary tumors and tumor metastasis in human pancreatic cancer. J Histochem Cytochem 49: 539-549, 2001.

18. Barrionuevo P, Beigier-Bompadre M, Ilarregui JM, Toscano MA, Bianco GA, Isturiz MA and Rabinovich GA: A novel function for galectin-1 at the crossroad of innate and adaptive immunity: galectin-1 regulates monocyte/macrophage physiology through a non-apoptotic ERK-dependent pathway. J Immunol 178: 436-445, 2007.
19. He J and Baum LG: Presentation of galectin-1 by extracellular matrix triggers $\mathrm{T}$ cell death. J Biol Chem 279: 4705-4712, 2004.

20. Hafer-Macko C, Pang M, Seilhamer JJ and Baum LG: Galectin-1 is expressed by thymic epithelial cells in myasthenia gravis. Glycoconj J 13: 591-597, 1996.

21. van den Brule F, Califice S, Garnier F, Fernandez PL, Berchuck A and Castronovo V: Galectin-1 accumulation in the ovary carcinoma peritumoral stroma is induced by ovary carcinoma cells and affects both cancer cell proliferation and adhesion to laminin-1 and fibronectin. Lab Invest 83: 377-386, 2003.

22. Moiseeva EV, Rapoport EM, Bovin NV, Miroshnikov AI, Chaadaeva AV, Krasilshschikova MS, Bojenko VK, Bijleveld C, van Dijk JE and Den Otter W: Galectins as markers of aggressiveness of mouse mammary carcinoma: towards a lectin target therapy of human breast cancer. Breast Cancer Res Treat 91: 227-241, 2005.

23. Kreunin P, Yoo C, Urquidi V, Lubman DM and Goodison S: Proteomic profiling identifies breast tumor metastasisassociated factors in an isogenic model. Proteomics 7: 299-312, 2007.

24. Jung EJ, Moon HG, Cho BI, Jeong CY, Joo YT, Lee YJ, Hong SC, Choi SK, Ha WS, Kim JW, Lee CW, Lee JS and Park ST: Galectin-1 expression in cancer-associated stromal cells correlates tumor invasiveness and tumor progression in breast cancer. Int J Cancer 120: 2331-2338, 2007.

25. Clausse N, van den Brule F, Waltregny D, Garnier F and Castronovo V: Galectin-1 expression in prostate tumorassociated capillary endothelial cells is increased by prostate carcinoma cells and modulates heterotypic cell-cell adhesion. Angiogenesis 3: 317-325, 1999.

26. Rao PS, Govindarajan R, Mallya KB, West W and Rao US: Characterization of a new antibody raised against the NH2 terminus of P-glycoprotein. Clin Cancer Res 11: 5833-5839, 2005.

27. Bidon-Wagner $\mathrm{N}$ and Le Pennec JP: Human galectin-8 isoforms and cancer. Glycoconj J 19: 557-563, 2004.

28. Zick Y, Eisenstein M, Goren RA, Hadari YR, Levy Y and Ronen D: Role of galectin-8 as a modulator of cell adhesion and cell growth. Glycoconj J 19: 517-526, 2004.

29. Rubinstein N, Ilarregui JM, Toscano MA and Rabinovich GA: The role of galectins in the initiation, amplification and resolution of the inflammatory response. Tissue Antigens 64: 1-12, 2004.

30. Druley TE, Stein WD and Roninson IB: Analysis of MDR1 Pglycoprotein conformational changes in permeabilized cells using differential immunoreactivity. Biochemistry 40: 4312-4322, 2001 .

31. Nuti SL, Mehdi A and Rao US: Activation of the human Pglycoprotein ATPase by trypsin. Biochemistry 39: 3424-3432, 2000.

32. Danguy A, Camby I and Kiss R: Galectins and cancer. Biochim Biophys Acta 1572: 285-293, 2002.

33. Gray CA, Dunlap KA, Burghardt RC and Spencer TE: Galectin15 in ovine uteroplacental tissues. Reproduction 130: 231-240, 2005.

34. Satterfield MC, Bazer FW and Spencer TE: Progesterone regulation of preimplantation conceptus growth and galectin 15 (LGALS15) in the ovine uterus. Biol Reprod 75: 289-296, 2006.

35. Lahm H, Andre S, Hoeflich A, Fischer JR, Sordat B, Kaltner H, Wolf $\mathrm{E}$ and Gabius $\mathrm{HJ}$ : Comprehensive galectin fingerprinting in a panel of 61 human tumor cell lines by RT-PCR and its implications for diagnostic and therapeutic procedures. J Cancer Res Clin Oncol 127: 375-386, 2001.

36. Miura T, Takahashi M, Horie H, Kurushima H, Tsuchimoto D, Sakumi K and Nakabeppu Y: Galectin-1beta, a natural monomeric form of galectin-1 lacking its six amino-terminal residues promotes axonal regeneration but not cell death. Cell Death Differ 11: 1076-1083, 2004. 